Gut, 1989, 30, 1401-1405

\title{
Comparison of albendazole, mebendazole and praziquantel chemotherapy of Echinococcus multilocularis in a gerbil model
}

\author{
D H TAYLOR, D L MORRIS, D REFFIN, AND K S RICHARDS \\ From the Department of Surgery, University Hospital, Nottingham; Department of Biological Sciences, \\ University of Keele, Keele, Staffs.
}

SUMmary The efficacy of albendazole $(50 \mathrm{mg} / \mathrm{kg} / \mathrm{d})$, mebendazole $(50 \mathrm{mg} / \mathrm{kg} / \mathrm{d})$ and praziquantel $(500 \mathrm{mg} / \mathrm{kg} / \mathrm{d})$ against established intraperitoneal infections of Echinococcus multilocularis in gerbils was compared by monitoring parasite weight and making ultrastructural observations on treated and untreated material. Praziquantel was the most active protoscolicidal agent, reducing protoscolex viability to $<2 \%$, although it did not inhibit cyst growth. Albendazole was the most effective agent in reducing cyst growth and was, when compared with other regimes significantly more effective than mebendazole $(p<0 \cdot 05)$, praziquantel $(\mathbf{p}<0 \cdot 01)$ or untreated controls $(\mathbf{p}<0 \cdot 01)$.

Many believe that the almost invariably fatal disease alveolar hydatidosis of man, caused by the cystic stage of Echinococcus multilocularis, is incurable. Not only is the resectability rate low but recurrence often occurs after seemingly successful surgical resection.'

$E$ multilocularis must not be confused with the much more benign $E$ granulosus responsible for cystic hydatid disease. E multilocularis is an infiltrative lesion which may look very similar to a hepatocellular carcinoma. This disease is very rare in the UK, but is seen in Central Europe (Switzerland, Northern France, Southern Germany, Austria, Northern Italy), the USSR, some parts of the Middle East, Japan, Alaska and the central United States. The life cycle includes fox as an intermediate host and microtine rodents are the commonest definitive host; a more dangerous domestic cat, dog/mouse cycle can occur. The terrifying problem of $E$ multilocularis is that there is no possible control mechanism - several countries have almost eliminated E granulosus (sadly not the UK) by dog control/regular deworming etc, but as both intermediate and definitive hosts are wild there is little prospect of controlling the spread of $E$ multilocularis.

Address for correspondence: D L. Morris. Department of Surgery. University Hospital. Nottingham. NG7 2UH.

Accepted for publication 14 February 1989.
For some years there has been sound experimental ${ }^{2.3}$ and increasing clinical evidence ${ }^{+x}$ that the benzimidazole carbamate, mebendazole, has at least a parasitostatic effect on E multilocularis. More recently experimental work using albendazole," another benzimidazole carbamate, and some encouraging preliminary clinical reports" using the same drug have raised hopes of a possible chemotherapeutic treatment for this disease.

The isoquinoline compound, praziquantel, has been shown to be extremely active against in vitro cultures of $E$ multilocularis protoscoleces and to achieve this effect more rapidly and at lower concentrations than benzimidazole carbamates. "We have also shown that praziquantel had some inhibitory effect on in vivo growth of this disease." The relative efficacies of these two drugs have not been compared with mebendazole, however, and the aim of the present study was therefore to examine the effect of mebendazole, albendazole, and praziquantel against E multilocularis infections in vivo.

Methods

ANIMALS

Fifty gerbils, which had three months previously received an intraperitoneal injection of $\simeq 4000$ live protoscoleces of $E$ multilocularis suspended in $0.5 \mathrm{ml}$ normal saline, were entered into the experiment. Ten 
of these animals were immediately killed to assess the percentage infection rate and the mean weight of parasite material at week zero. The remaining 40 animals were divided into groups of 10 and placed into one of four categories: controls, albendazole 50 $\mathrm{mg} / \mathrm{kg} / \mathrm{d}$, mebendazole $50 \mathrm{mg} / \mathrm{kg} / \mathrm{d}$, praziquantel 500 $\mathrm{mg} / \mathrm{kg} / \mathrm{d}$. Drugs were administered in a single daily $0.5 \mathrm{ml}$ dose by gavage and treatment continued five days a week for 25 weeks. Throughout the experimental period, the animals were weighed weekly, and after 25 weeks all the surviving animals underwent necropsy. All parasite material was then removed and weighed, and samples of protoscoleces from within cysts were taken for viability assessment by eosin exclusion and flame cell activity. Small portions of cyst material from peripheral areas of the infection were removed for electron microscopy and fixed in $3 \%$ glutaraldehyde in phosphate buffer $(\mathrm{pH}$ $7 \cdot 2$ ) for a minimum of six hours before a buffer wash and post-fixation in buffered $1 \%$ osmium tetroxide. After dehyration in an acetone series, the tissue was embedded in Spurr's resin. Routine $0.5 \mu \mathrm{m}$ sections were taken and stained with Toluidine blue before cutting ultrasections that were stained with aqueous uranyl acetate followed by lead citrate before examination.

\section{Results}

Of the 40 gerbils entered into the 25 week experiment, 22 survived and underwent necropsy. These consisted of: five of 10 controls; eight of 10 albendazole treated; five of 10 mebendazole treated; four of 10 praziquantel treated. Four of the mebendazole treated animals died within a week of beginning chemotherapy and may have been related to toxicity. All the remaining 14 animals which did not survive till the end of the experiment, died of very advanced abdominal disease, and it is noted that the survival rate was higher in the albendazole treated group.

The Table shows the weight of parasite material

Table Animal survival, mean parasite weight and protoscolex viability in albendazole, mebendazole and praziquantel treated gerbils, and control animals killed at week 0 and week 25 .

\begin{tabular}{|c|c|c|c|c|}
\hline & \multicolumn{2}{|c|}{$\begin{array}{l}\text { Parasite } \\
\text { weight mean } \\
(S E)(g)\end{array}$} & \multirow{3}{*}{ 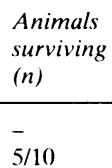 } & \multirow{2}{*}{$\begin{array}{l}\begin{array}{l}\text { Viability of } \\
\text { protoscoleces } \\
(\%)\end{array} \\
95 \%\end{array}$} \\
\hline (wk zero) & $5 \cdot 4$ & $(1 \cdot 4)$ & & \\
\hline$(w k 25)$ & $18 \cdot 5$ & $(4 \cdot 8)$ & & $95 \%$ \\
\hline Albendazole (wk 25) & $3 \cdot 78$ & $(1 \cdot 23)$ & $8 / 10$ & $25 \%$ \\
\hline Mebendazole (wk 25) & $9 \cdot 2$ & $(1 \cdot 74)$ & $5 / 10$ & $67 \%$ \\
\hline praziquantel ( $w k 25$ ) & $17 \cdot 2$ & $(0 \cdot 5)$ & $4 / 10$ & $<2 \%$ \\
\hline
\end{tabular}

and protoscolex viability in the 10 controls killed at week zero, and in the 22 killed at week 25 . Parasite weight in control and praziquantel treated animals was significantly more by week 25 than in the controls sacrificed at week zero $(p<0 \cdot 01)$. By week 25 in the mebendazole treated animals, parasite weight was more than that of controls at week zero, but not significantly so $(p<0 \cdot 1)$. Albendazole treatment achieved a small reduction in parasite weight when compared with controls killed at week zero, but this was not statistically significant.

When the effects of the chemotherapeutic agents are compared with the untreated control group killed at week 25 , the weight of parasite material removed from animals treated with albendazole was significantly less than that removed from untreated controls $(p<0.01)$ or from animals treated with mebendazole $(\mathrm{p}<0.05)$ or from praziquantel treated animals $(\mathrm{p}<0.001)$. The parasite material removed from the mebendazole treated animals was significantly less than that removed from praziquantel treated animals $(p<0.001)$, and although, when compared with untreated controls, there was a trend towards lower parasite weight in the mebendazole treated animals, this failed to achieve statistical significance. Praziquantel treatment appeared not to affect parasite weight.

Percentage viabilities of sampled protoscoleces as determined by eosin exclusion and flame cell activity are shown in the Table.

Figure 1 graphically represents the mean animal weight change of each experimental group from a starting weight expressed as zero. Mean animal weight gain between weeks 10 to 25 showed that

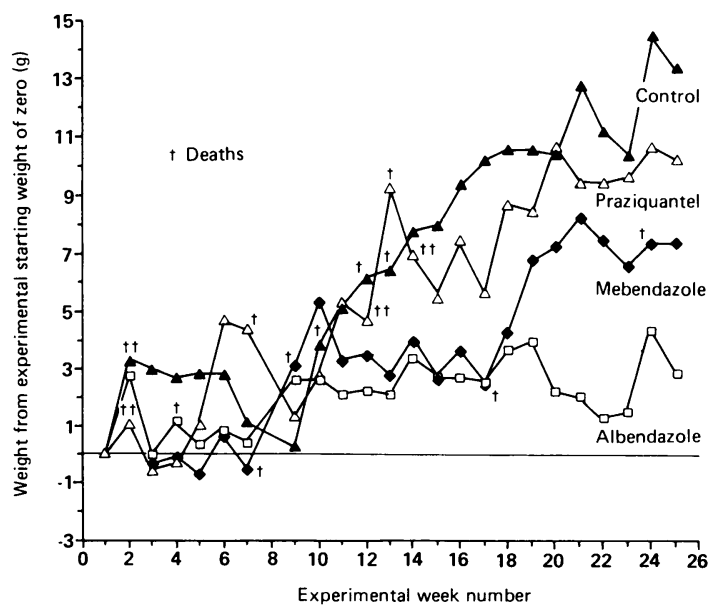

Fig. 1 Mean animals weight change from starting weight zero in control, mebendazole, albendazole and praziquantel treated animals. 
animals receiving albendazole gained significantly less weight than either the control, mebendazole treated or praziquantel treated groups $(p<0 \cdot 001)$. Mebendazole treated gerbils gained significantly less weight than the controls or the praziquantel treated animals $(p<0.01)$ whilst the weight gain within the praziquantel treated group was not significantly less than the controls, though a trend was apparent $(\mathrm{p}=0 \cdot 1)$.

It will be seen that the weight gains of differently treated groups of animals closely parallel the weight of parasite material recovered at necropsy. We have previously shown that chemotherapy has little if any effect on the weight of uninfected animals."

Ultrastructurally, the germinal layer (Fig. 2) and the tegument of protoscoleces (Fig. 3) of hydatids from untreated controls sacrificed at week 25 appeared normal, though occasionally a profile of a dead disintegrating protoscolex was observed, as is customary in established infections.

Material from mebendazole treated animals (Fig. 4) differed little from control tissue with the exception of increased vesiculation within the tegumentary cytons which occasionally possessed residual bodies. Brood capsule walls and protoscoleces appeared unaffected and the latter were similar to that illustrated in Fig. 3.

In albendazole treated tissue, the germinal layer varied from showing increased vesiculation similar to that seen in mebendazole treated material (Fig. 4) to loss of integrity (Fig. 5) with cyton fragments lying beneath a distal cytoplasm that, in places, was represented only by the truncated microtriches.
Frequently the germinal layer was present only as vesicles and fragmented profiles (Fig. 6), and the central cavity of such loculi contained vesicular masses and detached protoscolex microtriches. All the examined profiles of intact protoscoleces, however, appeared normal (Fig. 2), as did the walls of the brood capsules.

Cystic tissue from praziquantel treated animals also varied, often with one loculus appearing little affected apart from slight increased vesiculation and an adjacent loculus showing complete disintegration of the germinal layer (Fig. 7). Other profiles ranged from substantial loss of integrity of the germinal layer (Fig. 8), often with mitochondria with atypical cristae, to its total disintegration (Fig. 9), with only vesicles of the distal cytoplasm remaining in contact with the laminated layer. In all sections that passed through protoscoleces only necrotic tissue was observed.

\section{Discussion}

We have previously reported that both the active metabolite of albendazole, albendazole sulphoxide and praziquantel can readily enter the cysts of E mutilocularis ${ }^{9}$ and are active against the protoscolex in in vitro culture. ${ }^{11}$ We have also reported in vivo activity of albendazole against $E$ multilocularis in an animal model. ${ }^{9}$

The present study has produced information concerning the in vivo protoscolicidal action of albendazole and prazquantel after therapy, which concurs with the previous in vitro results in that praziquantel was significantly more effective than

Fig. 2 Control (week 25): intact germinal layer. D, distal cytoplasm; T, tegumentary cyton; $N, N u c l e u s ; G$, glycogen; L, laminated layer. Bar $=1 \mu \mathrm{m}$.

Fig. 3 Control protoscolex: Low powered micrograph of rostellar and sucker regions. $R$, lateral edge of rostellum; arrow, rostellar hook; $S$, scolex distal cytoplasm bearing microtriches (arrowheads); $T$, tegumentary cyton with nucleus $(N)$. Bar $=2$ $\mu \mathrm{m}$.

Fig. 4 Mebendazole: Intact germinal layer with vesiculated $(V)$ cytons. $D$, distal cytoplasm; $G$, glycogen; L, laminated layer. Bar $=I \mu m$.

Fig. 5 Albendazole: Disintegrated germinal layer. Arrows, distal cytoplasm eroded; $F$, tegumentary cyton fragments with vesicles $(V)$; L, laminated layer. Bar $=1 \mu \mathrm{m}$.

Fig. 6 Albendazole: germinal layer represented only by vesiculated cyton fragments $(F)$. L, laminated layer. Bar $=1 \mu m$.

Fig. 7 Praziquantel: two adjacent loculi; the left $(L t)$ with an intact germinal layer, the right $(R t)$ with only tissue fragments in contact with the laminated layer $(L)$. Bar $=1 \mu \mathrm{m}$.

Fig. 8 Praziquantel: the germinal layer has lost integrity; though truncated microtriches (arrows) still remain in contact with the laminated layer $(L)$. Cyton fragments contain mitochondria (double arrows) with atypical cristae. Bar $=1 \mu \mathrm{m}$.

Fig. 9 Praziquantel: germinal layer represented only by vesicles (arrows) of the distal cytoplasm. L, laminated layer. Bar $=I \mu m$. 

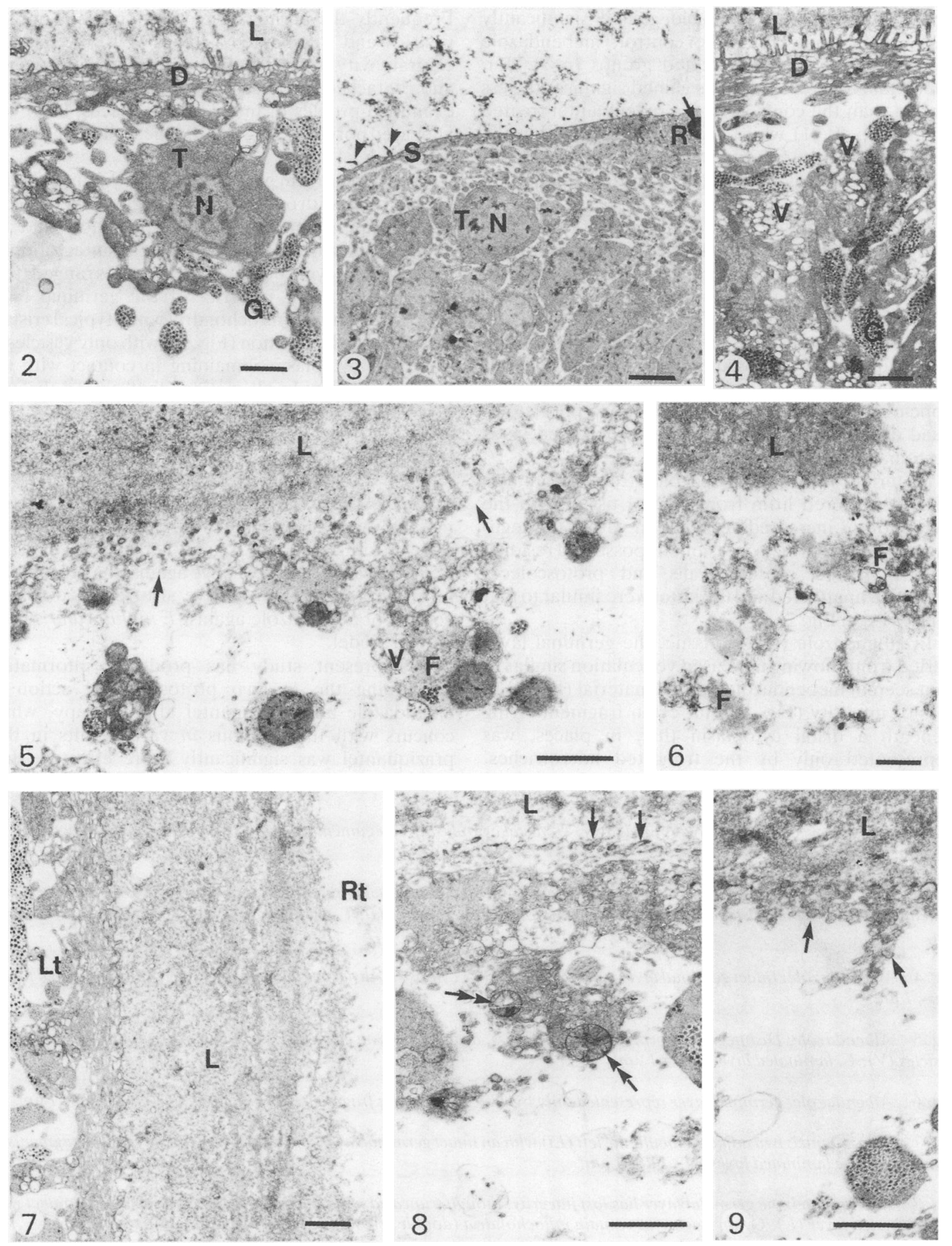
albendazole sulphoxide." In contrast, mebendazole appeared to have only a slight protoscolicidal action in this in vivo model, and the ultrastructural observations confirmed these results.

The efficacy of albendazole in reducing the rate of cyst growth, as measured by parasite weight, was greater than either mebendazole at the same dose or praziquantel at 10 times the dosage. Regression of established disease apparently occurred and animal survival was probably enhanced.

The ultrastructural study on albendazole and mebendazole treated tissue concurred with the results obtained by recording parasite weight, and the greater efficacy of albendazole was demonstrated. In contrast, praziquantel did not significantly reduce weight gain even at this high dose of $500 \mathrm{mg} / \mathrm{kg} / \mathrm{d}$ which is 10 times the dose used in man, yet the majority of profiles observed ultrastructurally showed either loss of integrity or near total disintegration in a manner similar to that reported for Egranulosus. ${ }^{2}$ Such tissue, although judged dead, would nevertheless contribute to parasite weight at necropsy.

These data are extremely encouraging, though we acknowledge that viable parasite material was present at the end of the experimental period, even in the albendazole treated animals, and this material almost certainly has the ability to resume growth. It is important, however, to note that the animals were treated only daily for five days per week by gavage, and better results might have been achieved using administration systems that would result in consistently higher serum concentrations.

It would, nevertheless, appear that the effect of albendazole is markedly parasitostatic in this gerbil model of established E multilocularis, and perhaps it should now be considered as the drug of choice in the treatment of this lethal disease in man. Whether praziquantel, with is undoubted ability to kill protoscolex, has a role in any clinical setting remains unclear. We have previously demonstrated that combination therapy is more effective than either agent alone for $E$ granulosus in both in vitro and in vilo systems ${ }^{13}$ and clearly this concept also deserves investigation in this lethal disease.
The financial support of Smith, Kline \& French (UK) and $\mathrm{E}$ Merck (Darmstadt) is gratefully acknowledged.

\section{References}

1 Mosimann F. Is alveolar hydatid discase of the liver incurable? Ann Surg 1980; 192: 118.

2 Eckert J. Burkhardt B. Chemotherapy of experimental echinococcosis. Acta Trop 1980; 37: 297-300.

3 Wilson JF, Rausch RL. Mebendazole and alveolar hydatid discase. Ann Trop Med Parasitol 1982: 76: 165-73.

4 Kern P. Human echinococcosis - follow up of 23 patients treatment with mebendazole. Infection 1983; 11: 17-30.

5 Luder PJ, Robitti G. Meisler P. Bircher J. High oral doses of mebendazole interfere with growth of larval Echinococcus multilocularis lesions. J Hepatol 1985; 1: 369-77.

6 Davis A. Pawlowski ZS. Dixon H. Multi-centre clinical trials of benzimidazole carbamates in human cchinococcosis. Bull WHO 1986; 64: 383-8.

7 Rausch RL, Wilson JF. McMahon BJ, O'Gorman MA. Consequences of continuous mebendazole therapy in alveolar hydatid discase with a summary of a 10 year clinical trial. Ann Trop Med Parasitol 1986; 80: 403-19.

8 Schroder R. Robotti G. New aspects in the management of alveolar echinococcosis involving the liver. World $J$ Surg 1986; 10: 968-73.

9 Taylor DH, Morris DL, Richards KS, Reffin D. Echinococcus multilocularis: in vivo results of therapy with albendazole and praziquantel. Trans $R$ Soc Med Hyg 1988; 82: 611-5.

10 Wilson JF, Rausch RL, McMahon BJ, Schantz PM, Trujillo DE, O'Gorman MA. Albendazole in alveolar hydatid disease. Am J Trop Med Hyg 1987; 37:162-8.

11 Taylor DH, Morris DL. In vitro culture of Echinococcus multilocularis: protoscolicidal action of praziquantel and albendazole sulphoxide. Trans $R$ Soc Trop Med Hyg $1988 ; 82 ; 26.5-7$.

12 Richards KS, Morris DL, Danicls D, Rilcy EM. Echinococcus granulosus; the effects of praziquantel, in vivo and in vitro, on the ultrastructure of equine strain murine cysts. Parasitology 1988; 96: 323-36.

13 Taylor DH, Morris DL, Richards KS. Combination chemotherapy in hydatid disease. Trans $R$ Soc Trop Med Hyg 1988; 82; 265-7. 